\title{
Implications of SNPs on toll-like receptor genes in malaria: what do we know?
}

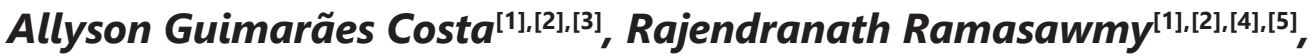 \\ Adriana Malheiro ${ }^{[1],[3],[5]}$ and Marcus Vinícius Guimarães Lacerda ${ }^{[1],[2],[6]}$
}

\begin{abstract}
[1]. Programa de Pós-Graduação em Medicina Tropical, Universidade do Estado do Amazonas, Manaus, AM, Brasil. [2]. Fundação de Medicina Tropical Dr. Heitor Vieira Dourado, Manaus, AM, Brasil. [3]. Laboratório de Genômica, Fundação Hospitalar de Hematologia e Hemoterapia do Amazonas, Manaus, AM, Brasil. [4]. Universidade Nilton Lins, Manaus, AM, Brasil. [5]. Programa de Pós-Graduação em Imunologia Básica e Aplicada, Universidade Federal do Amazonas, Manaus, AM, Brasil. [6]. Instituto Leônidas e Maria Deane, Fundação Oswaldo Cruz, Manaus, AM, Brasil.
\end{abstract}

Human host immune response to malaria infection is triggered by the innate immune system that shapes adaptive immunity. Toll-like receptors (TLRs), one of the pattern recognition receptors (PRRs) of the innate immune system, recognize several components of Plasmodium sp., described as pathogen associated molecular patterns (PAMPs) ${ }^{1}$.

Glycosylphosphatidylinositol-anchored (GPI), merozoite surface proteins (MSP1 and MSP2), microparticles, hemozoin, and parasite deoxyribonucleic acid (DNA) are TLR ligands. TLR-2, TLR-4, and TLR-9 are responsible for the recognition of these components to trigger a cascade of reactions leading to the liberation of the transcription factor NF- $\mathrm{KB}$ in the cytoplasm to enter the nucleus and transcribe inflammatory mediators ${ }^{2}$.

The immune system has been shaped by the process of evolution into an efficient system that allows the coexistence of pathogenic agents in hosts while controlling infections ${ }^{3}$. Knockout murine models of genes involved in the innate immune response have shown the key role played by TLRs in Plasmodium spp. infection ${ }^{4}$.

In humans, Plasmodium infection results in a wide spectrum of clinical manifestations that depend mostly on the interaction between the vector-parasite-host, associated environmental factors, parasite load, immunological status, and host genetic background (Figure 1) $)^{5}$.

Several single nucleotide polymorphisms (SNPs) in the $T L R$ genes are associated with infectious diseases. Some of these single base pair changes, called point mutations, are non-synonymous, causing an amino acid exchange in the gene and others in the promoter regions influence the expression of the receptors ${ }^{6}$.

The study by Dhangadamajhi et al. ${ }^{7}$ published in this issue of the Journal of the Brazilian Society of Tropical Medicine entitled "The meta-analysis of TLR4 and TLR9 SNPs implicated

Corresponding author: Dr. Marcus Vinicius Guimarães de Lacerda. e-mail: marcuslacerda.br@gmail.com

Received 30 March 2017

Accepted 6 April 2017 in severe malaria" highlights the importance of the SNPs in TLR4 (Asp299Gly) and TLR9 (-1237C/T and -1486C/T) in malaria. This meta-analysis study suggests the implications of these TLR polymorphisms on the pathogenesis of malaria and the association with severe clinical manifestations of the disease.

Several studies have reported controversial results about the association of these genetic variants with Plasmodium falciparum infection. Here, the authors point out that the differences in the design of the studies, ethnic structure of the population, and methodologies applied make it difficult to compare the studies. This holds truth with respect to the study design and population ethnicities. However, allelic discrimination by any genotyping technology should not influence the allele frequencies observed in any study, especially in research laboratories. As suggested by the authors, selecting healthy controls for case-control studies in genetic infectious diseases is always problematic. However, asymptomatic individuals infected with the parasite will always be the best control, especially when they have never experienced any clinical manifestation.

The authors noted an association of the variants $-1486 C / T$ and $-1237 C / T$ of $T L R 9$ with severe malaria by using conventional and stratified analysis models for a total of 665 patients with malaria and 1,187 individuals with uncomplicated malaria. Only five studies were included, two from India and three from Africa. This meta-analysis study has its merits; however, the sample size is still quite small. Unfortunately, in the Indian studies, both variants of TLR9 are not in Hardy-Weinberg equilibrium and this may skew any association.

In Brazil, polymorphisms in TLR4 and TLR9 are described to be associated with malaria susceptibility and with increased parasitemia in P. falciparum infection or mixed infections ${ }^{8,910}$. These studies are not included in the meta-analysis presented in this issue. However, these studies from Latin America are important as they provide the allelic frequencies of each SNP in addition to their associations with parasitemia.

Altogether, this meta-analysis study indicates the implications of these TLR SNPs, which may be in part responsible for inter-individual variation in severe clinical manifestation of the disease. Studies of these SNPs using a large sample size 


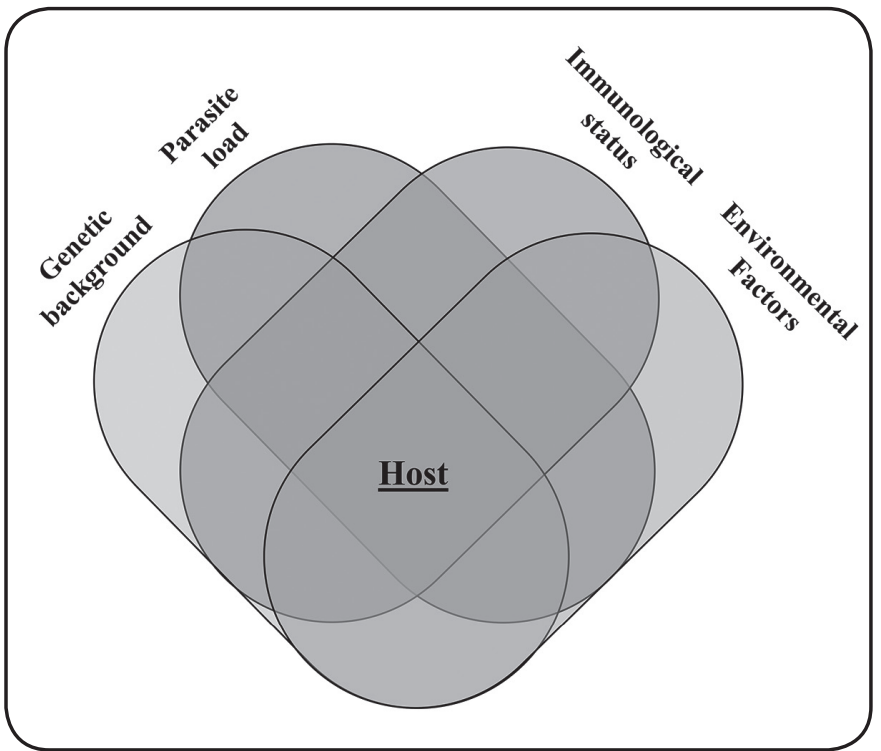

FIGURE 1 - Venn diagram of the characteristics involved in pathogenesis and severe clinical manifestations of the Plasmodium sp. infection.

of individuals with uncomplicated, asymptomatic, or severe malaria may contribute to the development and practical application of an effective vaccine against the parasite and in the design of tools for disease control and elimination.

\section{Conflicts of interests}

The authors declare that there is no conflict of interest.

\section{REFERENCES}

1. Kawai T, Akira S. Toll-like receptors and their crosstalk with other innate receptors in infection and immunity. Immunity. 2011;34(5):637-50.

2. Gazzinelli RT, Kalantari P, Fitzgerald K a, Golenbock DT. Innate sensing of malaria parasites. Nat Rev Immunol. 2014;14(11):744-57.

3. Bozza MT, Martins YC, Carneiro L a M, Paiva CN. Macrophage migration inhibitory factor in protozoan infections. J Parasitol Res. 2012;2012:413052.

4. Coban C, Ishii KJ, Horii T, Akira S. Manipulation of host innate immune responses by the malaria parasite. Trends Microbiol. 2007;15(6):271-8.

5. Mackinnon MJ, Mwangi TW, Snow RW, Marsh K, Williams TN. Heritability of malaria in Africa. PLoS Med. 2005;2(12):1253-9.

6. Schröder NWJ, Schumann RR. Single nucleotide polymorphisms of Toll-like receptors and susceptibility to infectious disease. Lancet Infect Dis. 2005;5(3):156-64.

7. Dhangadamajhi G, Kar A, Rout R, Majhi PD. A meta-analysis of TLR4 and TLR9 SNPs implicated in severe malaria. Rev Soc Bras Med Trop. 2017;50(2):153-160.

8. Soares SC, Abé-sandes K, Nascimento-Filho VB, Nunes FMF, Silva-Jr WA. Genetic polymorphisms in TLR4, CR1 and Duffy genes are not associated with malaria resistance in patients from Baixo Amazonas region, Brazil. Genet Mol Res. 2008;7(4):1011-9.

9. Leoratti FMS, Farias L, Alves FP, Suarez-Mútis MC, Coura JR, Kalil J, et al. Variants in the toll-like receptor signaling pathway and clinical outcomes of malaria. J Infect Dis. 2008;198(5):772-80.

10. da Silva Santos S, Clark TG, Campino S, Suarez-Mutis MC, Rockett KA, Kwiatkowski DP, et al. Investigation of Host Candidate Malaria-Associated Risk/Protective SNPs in a Brazilian Amazonian Population. PLoS One. 2012;7(5):e36692. 